than 40 years underwent uricemia testing (HumaSens). Gout was defined by a validated algorithm (1). Hyperuricemia was defined by capillary level equivalent to plasma uric acid level (PUA) $>6 \mathrm{mg} / \mathrm{dl}$ (2) and/or urate lowering drug (ULD) prescription.

Results: 1.144 participants (mean age 37.7 years; $50.4 \%$ men) were included. Prevalence of gout in the entire redressed sample was $3.3 \%(95 \% \mathrm{Cl} 2.2-4.9)$. The prevalence was $4.1 \%(1.8-8.9), 2.6 \%(1.4-4.7), 6.7 \%(2.5-16.8)$, and $1.9 \%(0.5-6.6)$ for Europeans, Melanesians, Polynesians and other ethnicities, respectively. After adjustment for age and sex, Polynesians showed higher risk for gout than Europeans (adjusted odds ratio [aOR] 4.57 [95\% Cl 1.3-16.7]). Prevalence of hyperuricemia, determined in 658 participants, was $67.0 \%(95 \% \mathrm{Cl}$ 61.9-71.6). Prevalence of hyperuricemia was greater for Polynesians (aOR 9.17 [3.2-26.4]), Melanesians (aOR 4.02 [2.2-7.2]) and other ethnicities (aOR 2.18 [1.1-4.5]) than Europeans. On univariate analysis, factors associated with gout were hyperuricemia, male gender, age, BMI, waist circumference, renal failure, hypertension, diabetes, history of major episodes of depression and cancer but not dietary factors or physical activity, despite a consistent association with BMI. Among gout and non-gout patients, $45.9 \%$ and $0.7 \%$ were receiving ULT. Overall, $29.6 \%$ of patients receiving ULD had proper control of PUA levels $(\leq 6 \mathrm{mg} / \mathrm{dl})$. Conclusions: As compared with Europe, in New Caledonia, the prevalence of gout and hyperuricemia was high, including in patients with European descent, as was previously reported for New Zealand (3). The prevalence of gout and hyperuricemia differed by ethnicity. For Melanesians, the prevalence of hyperuricemia was higher but risk of gout similar to that for Europeans, so factors (e.g., genetics) other than those involved in hyperuricemia may intervene in the risk of gout.

References:

[1] Richette et al. Ann Rheum Dis 2015;74:1684-90.

[2] Fabre et al ACR meeting, Washington 2016.

[3] Winnard et al Rheumatology 2012;51:901-9.

Disclosure of Interest: None declared

DOI: 10.1136/annrheumdis-2017-eular.3409

\section{THU0464 A GENOME-WIDE ASSOCIATION STUDY OF GOUT IN PEOPLE OF EUROPEAN ANCESTRY}

T.R. Merriman ${ }^{1}$, M. Cadzow ${ }^{1}$, M. Merriman ${ }^{1}$, A. Phipps-Green ${ }^{1}$, R. Topless ${ }^{1}$, A. Abhishek ${ }^{2}$, M. Andres ${ }^{3}$, L. Bradbury ${ }^{4}$, R. Buchanan ${ }^{5}$, K. Cremin ${ }^{6}$, E. de Guzman $^{4}$, J. de Zoysa ${ }^{7}$, M. Doherty ${ }^{2}$, C. Hill ${ }^{8}$, T. Huizinga ${ }^{9}$, T. Jansen ${ }^{10}$, M. Janssen ${ }^{11}$, L. Joosten ${ }^{12}$, F. Kurreeman ${ }^{9}$, S. Lester ${ }^{8}$, F. Liote ${ }^{13}$, D. Macartney-Coxson ${ }^{14}$, H. Matsuo ${ }^{15}$, G. McCarthy ${ }^{16}$, S. McCormick $^{1}$, R. Murphy ${ }^{7}$, K. Pavelka ${ }^{17}$, F. Perez-Ruiz ${ }^{18}$, J. Puig ${ }^{19}$, T. Radstake 20 , P. Riches ${ }^{21}$, M. Rischmueller ${ }^{8}$, E. Roddy ${ }^{22}$, M. Smith ${ }^{23}$, E. Stahl ${ }^{24}$ B. Stiburkova ${ }^{17}$, R. Stubbs ${ }^{25}$, A.-K. Tausche ${ }^{26}$, R. Torres ${ }^{19}$, R. Walker ${ }^{1}$, K. Yamamoto ${ }^{27}$, M. Brown ${ }^{4}$, H. Choi ${ }^{28}$, N. Dalbeth ${ }^{7}$, A. So ${ }^{29}$, L. Stamp ${ }^{1}$, T. Flynn ${ }^{1} .{ }^{1}$ University of Otago, Dunedin, New Zealand; ${ }^{2}$ University of Nottingham, Nottingham, United Kingdom; ${ }^{3}$ Universidad Miguel Hernández, Alicante, Spain; ${ }^{4}$ Queensland University of Technology, Brisbane; ${ }^{5}$ University of Melbourne, Melbourne; ${ }^{6}$ University of Queensland, Brisbane, Australia; ${ }^{7}$ University of Auckland, Auckland, New Zealand; ${ }^{8}$ Queen Elizabeth Hospital, Adelaide, Australia; ${ }^{9}$ Leiden University Medical Center, Leiden; ${ }^{10}$ VieCuri Medical Centre, Venlo; ${ }^{11}$ Rijnstate Hospital, Arnhem; ${ }^{12}$ Radboud University Medical Center, Nijmegen, Netherlands: ${ }^{13}$ Hospital Lariboisière, Paris. France; ${ }^{14}$ Environmental and Scientific Research, Wellington, New Zealand; ${ }^{15}$ National Defense Medical College, Saitama, Japan; ${ }^{16}$ University College Dublin, Dublin, Ireland; ${ }^{17}$ institute of Rheumatology, Prague, Czech Republic; ${ }^{18} \mathrm{Hospital}$ de Cruces, Vizcaya; ${ }^{19}$ Hospital Universitario la Paz, Madrid, Spain; ${ }^{20}$ University Medical Centre Utrecht, Utrecht, Netherlands; ${ }^{21}$ University of Edinburgh, Edinburgh; ${ }^{22}$ Keele University, Newcastle, United Kingdom; ${ }^{23}$ Flinders Medical Centre and Repatriation Hospita, Adelaide, Australia; ${ }^{24} \mathrm{Mt}$ Sinai School of Medicine, New York, United States; ${ }^{25} \mathrm{P} 3$ Research Ltd, Wellington, New Zealand; ${ }^{26}$ University of Dresden, Dresden, Germany; ${ }^{27}$ Kurume University, Kurume, Japan; ${ }^{28}$ Harvard Medical School, Boston, United States; ${ }^{29}$ University of Lausanne, Lausanne, Switzerland

Background: Gout progresses through three stages: hyperuricemia, deposition of monosodium urate (MSU) crystals, and innate immune system response to MSU crystals. Genome wide association studies (GWAS) have provided insight into the molecular control of progression to hyperuricemia. However, less is known about the progression from hyperuricemia to gout.

Objectives: To conduct a GWAS for gout (where an immune response to MSU crystals has occurred) using 5,835 cases - the largest GWAS of gout to date.

Methods: The GWAS comprised 3 data sets: NZ/Eurogout (2,365 clinicallyascertained cases; 1,485 controls), the Health Professionals Follow-Up (HPFS) and Nurses' Health Studies (NHS) (1,038 cases, self-ascertained using ACR criteria; 1,095 controls), and UK Biobank (2,432 cases, ascertained by self-report of gout, hospital records, and/or use of urate-lowering therapy; 102,989 controls). The NZ/Eurogout samples were genotyped using the Illumina CoreExome v24 bead chip array (547,644 markers), the HPFS/NHS samples using the Illumina OmniExpress v12 bead chip array (730,525 markers), and the UK Biobank samples using an Affymetrix Axiom array (820,967 markers). UK Biobank genotypes had been imputed to $733.3 \mathrm{M}$ SNPs. Neither the NZ/Eurogout nor NHS/HPFS genotype sets were imputed. Markers common to all three data sets
$(279,939)$ were associated with gout (adjusted for sex, age) within each data set separately using PLINK 1.9. An inverse-variance weighted meta-analysis was done with meta v4.4 in R.

Results: There were seven loci with genome-wide significant $\left(P<5 \times 10^{-8}\right)$ evidence for association with gout: SLC2A9 (OR=1.67), ABCG2(OR=1.72), GCKR (OR=1.24), SLC17A1-A4 (OR=1.20), SLC22A12 (OR=1.21), PDZK1 (OR=1.14), TRIM46 (OR=1.18).

Conclusions: All seven loci have been previously associated with serum urate levels in GWAS. Our data emphasise the relative importance of genetic control of serum urate, compared to the genetic control of MSU crystal formation or the innate immune response, in determining gout.

Disclosure of Interest: None declared

DOI: 10.1136/annrheumdis-2017-eular.5634

\section{THU0465 CALCIUM PYROPHOSPHATE DEPOSITION DISEASE AND OSTEOARTHRITIS: TWO FACES OF THE SAME MEDAL? AN ULTRASONOGRAPHIC AND MICROSCOPY STUDY}

V. Picerno ${ }^{1}$, A. Scanu ${ }^{2}$, A. Adinolfi ${ }^{1}$, C.A. Scirè ${ }^{3}$, B. Frediani ${ }^{1}$, L. Punzi ${ }^{2}$, G. Filippou ${ }^{1} .{ }^{1}$ University of Siena, Siena; ${ }^{2}$ University of Padua, Padua; ${ }^{3}$ University of Ferrara, Ferrara, Italy

Background: Calcium pyrophosphate deposition disease (CPPD) and Osteoarthritis $(\mathrm{OA})$ are frequently associated and CPPD with OA is recognized as a clinical subtype [1]. However, the differences in pathogenetic, microscopic and clinical aspects between the two diseases are not clear and how CPPD and OA could affect each other is still a matter of debate.

Objectives: To assess the differences between CPPD and OA in terms of anatomic alterations of the joint, evaluated with US, and characteristics of the synovial fluid (SF) of knees affected by CPPD and/or OA.

Methods: consecutive patients reaching the outpatient clinic for the presence of knee pain and with any amount of joint effusion were eligible for the study. Patients with diagnosis or suspicion of chronic inflammatory rheumatic conditions were excluded. All enrolled patients underwent US of the knee for the assessment of joint effusion (JE), synovial hypertrophy (SH), synovial power Doppler (PD), femuro-tibial osteophytes (FTO) and alterations of the femoral hyaline cartilage (FHC), using a semiquantitative score (0: normal to 3 : severe alteration) and finally a US guided aspiration of the SF. SF was examined by optical and polarized light microscopy to determine total and differential white blood cell (WBC) counts, and for crystal identification. The concentration of the main inorganic ions involved in CPP crystal formation $\left(\mathrm{P}_{2} \mathrm{O}_{-}^{4-}, \mathrm{PO}_{4}^{3-}, \mathrm{Ca}^{2+}\right.$ and $\left.\mathrm{Mg}^{2+}\right)$ was assessed by fluorometric and colorimetric assays. CPP crystal detection was also used for the classification of patients. Further, in CPPD patients, a count of the CPP crystals detectable in a single slide was carried out. Depending on the variables, chi-square, Mann Whitney and Spearman Ro tests were used for statistical analysis.

Results: 49 patients ( 28 women), mean age 70.29 yo $(S D \pm 10.93)$ were enrolled in the study; 23 subjects presented OA and 26 CPPD (23.07\% acute arthritis, $77.6 \%$ CPPD with OA). At US, a statistically significant difference between CPPD and OA was found only for the grade of effusion, being more abundant in CPPD patients. On the contrary, no differences were found regarding SH, PD, FTO, FHC. SF analysis showed that CPPD patients presented a higher volume of SF, a higher total WBC count with a higher polymorphonuclear (PMN) cells percentage and lower monocytes percentage than patients with OA. Further, both total cell count and PMN percentage were positively correlated with the number of crystals in the SF. On the other hand, no statistically significant differences were found in the content of inorganic ions between the two groups.

Conclusions: According to these results, patients with CPPD and OA present some distinct features, mainly regarding the characteristics of the SF, compared to patients with $\mathrm{OA}$ alone. These differences may reflect different underlying pathogenetic pathways for the two diseases. Surprisingly, the concentration of inorganic ions in the two populations was similar.Further studies are necessary in order to better understand the link between CPPD and OA and the role of ions concentration in the SF for the formation of crystals.

References:

[1] Zhang W et al. European League Against Rheumatism reccomandations for calcium pyrophosphate deposition. Pat I: terminology and diagnosis. Ann Rheum Dis 2011; 70: 563-570.

Disclosure of Interest: None declared

DOI: 10.1136/annrheumdis-2017-eular.6606

\section{THU0467 SAFETY AND EFFICACY OF FEBUXOSTAT IN ADVANCED CKD PATIENTS WITH HYPERURICEMIA}

Y.-J. Oh, E.S. Park, S.S. Ahn, K.Y. Lee, S.-J. Byun, J.Y. Pyo, S.M. Jung, S.-W. Lee, Y.-B. Park, J.J. Song. Internal medicine, Yonsei University College of Medicine, Seoul, Korea, Republic Of

Background: In chronic kidney disease (CKD) patients, hyperuricemia is a common finding and might be one of modifiable risk factors for renal progression. However, dosing adjustments and increased risk of serious side effects of uric acid lowering agents in patients with reduced renal function lead to undercorrection of hyperuricemia, especially in patients with advanced CKD. Febuxostat is highly 